\title{
Direct Growth of Enclosed ZnO Nanotubes
}

\author{
Bin Liu' and Hua Chun Zeng ${ }^{1,2}(\bowtie)$ \\ ${ }^{1}$ Department of Chemical and Biomolecular Engineering, ${ }^{2}$ Minerals, Metals, and Materials Technology Center (M3TC), Faculty of \\ Engineering, National University of Singapore, 10 Kent Ridge Crescent, Singapore 119260
}

Received: 23 December 2008/Revised: 30 December 2008 / Accepted: 1 January 2009

(C)Tsinghua University Press and Springer-Verlag 2009. This article is published with open access at Springerlink.com

\begin{abstract}
To date, wet syntheses of single-crystalline $\mathrm{ZnO}$ micro- and nanotubes have been carried out using a twostep indirect approach in which a selective dissolution step is required in order to create the vacant space in the tubular structures. In this work, we develop a direct growth process for preparation of single-crystal $\mathrm{ZnO}$ nanotubes and nanorods. We also report that a concave shaped crystal growth front is generally reactive and offers a large surface area for matter deposition during rapid expansion of unidirectional nanomaterials. Depending on the degree of supersaturation of nutrients in solution, the concave growth front can either remain unaltered or undergo a concave-to-convex transformation, leading to the growth of solid nanorods and/or hollow nanotubes. The observed volume inversion should, in principle, also be applicable to the nanoarchitecture of other one-dimensional wurtzite structured nanomaterials, although individual sets of synthesis parameters need to be developed for each target material.
\end{abstract}

\section{KEYWORDS}

Nanotubes, zinc oxide, crystal grwoth

\section{Introduction}

Nanostructured tubular materials (or micro-, and nanotubes) have been extensively investigated for almost two decades [1-20]. In addition to carbon nanotubes first reported in 1991 [1], a great number of inorganic tubular microstructures and nanostructures have also been designed and prepared [1-20]. Structurally, these inorganic tubes can be broadly divided into two basic classes: (i) sheet type for layered materials [1-9], and (ii) monocrystalline or polycrystalline type for nonlayered materials [10-20]. Type (i) materials are also called inorganic fullerene-like micro- and nanotubes in which inorganic "molecular" layers are assembled into concentric cylinders or rolled into tube-like structures, while Type (ii) materials are essentially pristine one-dimensional monocrystalline or polycrystalline materials but with an additional central vacant space.

Among the various inorganic tubular materials, microtubes and nanotubes of zinc oxide $(\mathrm{ZnO})$ have received great attention [10-20] because this oxide semiconductor has many outstanding properties (e.g., $\mathrm{ZnO}$ has a direct band gap energy of $3.37 \mathrm{eV}$ and an exciton binding-energy of $60 \mathrm{meV}$ ) and has shown promising potential in various applications [21 -27]. Three different approaches have been reported

Address correspondence to chezhc@nus.edu.sg 
for the fabrication of $\mathrm{ZnO}$ tubular structures. The first one is solution synthesis [10-14]. This approach normally involves a two-step process in which solid forms of one-dimensional $\mathrm{ZnO}$ are first formed, and tubular structures are then obtained by dissolution of materials from the axial part of the solid structures [10 -14]. The dissolved axial part materials can be either $\mathrm{ZnO}$ itself or other templating materials [11]. The second approach requires a template, in which $\mathrm{ZnO}$ is first formed on the external surfaces of metallic zinc nanowires and nanorods, and the removal of metallic cores into the vapor phase at elevated temperatures subsequently gives rise to tubular structures of $\mathrm{ZnO}[15,16]$. Understandably, the hollow $\mathrm{ZnO}$ structures prepared by this method are likely to be polycrystalline $[15,16]$. The third strategy involves vapor phase deposition and/or reactions that can be carried out with pulsed laser deposition (PLD), chemical vapor deposition (CVD) and metalorganic chemical vapor deposition (MOCVD), by which open ended $\mathrm{ZnO}$ nanotubes can be prepared directly or indirectly [17-20].

Wet synthesis is in general more attractive compared to the other two dry approaches, because it is able to produce high quality monocrystalline $\mathrm{ZnO}$ micro- and nanotubes at low cost, and thus promises large-scale production of novel $\mathrm{ZnO}$ materials. Apart from the indirect creation of interior spaces via selective dissolution for $\mathrm{ZnO}$ micro- and nanotubes as reported earlier [10-14], the search for other newer methods such as preparation of singlecrystal $\mathrm{ZnO}$ nanotubes through direct crystal growth in solution, i. e., similar to those grown in the vapor phase [17-20] represents a new challenge in this field of research and should be further investigated. In this article, therefore, we explore a synthetic approach for direct growth of single-crystalline $\mathrm{ZnO}$ nanotubes. Using our approach, the central vacant space of $\mathrm{ZnO}$ nanotubes can be created through crystal growth instead of the reported dissolution processes. This direct growth method leads to formation of enclosed single-crystalline $\mathrm{ZnO}$ nanotubes with uniform diameters.

\section{Experimental}

Precursor solution preparation. $80 \mathrm{~g}$ or $40 \mathrm{~g}$ of $\mathrm{NaOH}$ pellets (99\%, Merck) and $29.748 \mathrm{~g}$ of $\mathrm{Zn}\left(\mathrm{NO}_{3}\right)_{2} \cdot 6 \mathrm{H}_{2} \mathrm{O}$ (98\%, Riedel-de Haen) were dissolved in deionized water $(200 \mathrm{~mL})$ to prepare a precursor solution with a molar ratio of $\mathrm{Zn}^{2+}: \mathrm{OH}^{-}$of 1:10 or 1:20, respectively. After dissolution of the two components, a clear solution was obtained.

Synthesis of $50 \mathrm{~nm} \mathrm{ZnO}$ nanorods with tubular growing heads. $3 \mathrm{~mL}$ of the above solution $\left(\mathrm{Zn}^{2+}\right.$ : $\left.\mathrm{OH}^{-}=1: 20\right)$ was mixed with $5 \mathrm{~mL}$ of deionized water. Then $25 \mathrm{~mL}$ of pure ethanol was added, followed by $5 \mathrm{~mL}$ of ethylenediamine (EDA; 99\%, Merck). The mixture was then kept in an ultrasonic water bath for 30-40 $\mathrm{min}$ and subsequently transferred to a Teflon-lined stainless steel autoclave. The autoclave was kept inside an electric oven set at $180{ }^{\circ} \mathrm{C}$ for $2 \mathrm{~h}$. After reaction, the autoclave was cooled under flowing water. The precipitate was separated by centrifugation and washed several times with deionized water and pure ethanol. The collected product was dried inside a vacuum desiccator at room temperature overnight.

Synthesis of $400 \mathrm{~nm} \mathrm{ZnO}$ nanotubes and nanorods. $3 \mathrm{~mL}$ of above solution $\left(\mathrm{Zn}^{2+}: \mathrm{OH}^{-}=1: 10\right)$ was mixed with $5 \mathrm{~mL}$ of deionized water. Then $30 \mathrm{~mL}$ of pure ethanol was added, followed by 5 $\mathrm{mL}$ of EDA ( $99 \%$, Merck). The mixture was then kept in an ultrasonic water bath for 20-40 min and subsequently transferred to a Teflon-lined stainless steel autoclave. The autoclave was kept inside an electric oven maintained at $180{ }^{\circ} \mathrm{C}$ for $2 \mathrm{~h}$. After reaction, the autoclave was cooled by running water. The precipitate was washed several times with deionized water and pure ethanol and harvested by centrifugation. The collected product was dried inside a vacuum desiccator at room temperature overnight.

Materials characterization. The structure of the $\mathrm{ZnO}$ products was characterized by powder X-ray diffraction (XRD; Shimadzu XRD-6000, Cu K $\alpha$ radiation). The size, morphology, and composition were measured by scanning electron microscopy (SEM, JSM-5600LV), transmission electron microscopy and selected-area electron diffraction (TEM/SAED; 
JEM-2010, $200 \mathrm{kV}$ ), and high-resolution TEM and energy dispersive X-ray spectroscopy (HRTEM/EDX; Philips-CM200 FEG, 200 kV). For sample preparation, a small drop of the $\mathrm{ZnO}$ rods/tubes redispersed by acetone was deposited on a copper grid (precoated with a carbon film) and dried in laboratory air.

\section{Results and Discussion}

Figure 1 describes schematically the two growth processes for the formation of the nanotubes and nanorods of $\mathrm{ZnO}$ obtained in our study. In route (a), a single crystal nucleus grows preferentially along its [0001] direction (stages i to iii; our current synthetic conditions favor the growth of [0001]). At a certain stage (stage iv), the wall rim grows faster than its central base, and a concave growth head is formed along the [0001] direction. When the reaction is accelerated after an induction period, rapid deposition of product species on the wall rims is expected, which results in a tubular interior (stage v); note that the six prismatic crystal planes of $\mathrm{ZnO}$ are fairly stable in this type of 1-D growth. When the nutrients are further consumed, the growth goes back to normal planar growth of $\{0001\}$, leading to an enclosure process for the nanotube, since the $\{0001\}$ in-plane growth will reduce the opening of the tubular structure. Under lower supersaturation conditions (stage vi; i.e., close to the end of the batch reactions), areas of the $\{0001\}$ planes are becoming smaller and smaller, resulting in a gradual reduction in head diameter. Associated with this process, from stages iv to vi, a growing nanotube head undergoes a shape transformation from a concave structure to a convex tip, that is, we observe an inversion from a "negative volume" (i.e., hollow space) to a "positive volume" (i.e., solid matter). In contrast to route (a), which creates kinetically an interior space within the 1-D structure, route (b) produces a solid nanorod. Although in stages iv and $\mathrm{v}$, a concave growing head can still be formed, the difference between the wall rim and central base is much smaller. This shallow vacant space can be readily filled up when the growth rate is slowed down, producing the solid nanorod. In both cases, however, the shapes of growing heads of the 1-D nanostructures undergo the same concave-to-

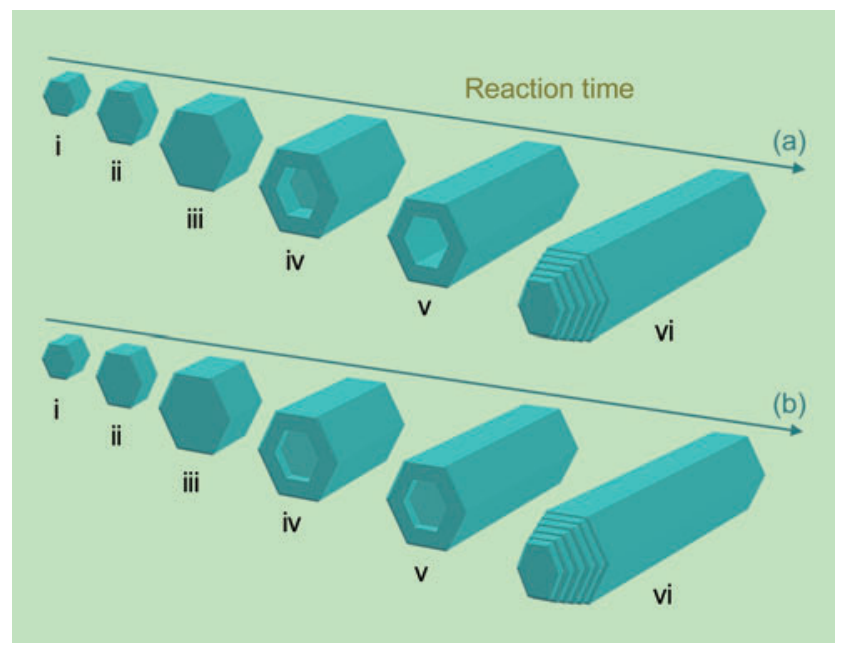

Figure 1 Schematic illustration of the formation of (a) $\mathrm{ZnO}$ nanotubes, and (b) ZnO nanorods, depending on relative growth rates of the wall rim and the central base of the growing heads. In both cases, the volume inversion of the growing head (along the [0001] direction) is depicted in stages iv-vi

convex transformation.

In the present work, we first employed a high alkali concentration (molar ratio of $\mathrm{Zn}^{2+} / \mathrm{OH}^{-}=1: 20$, see Experimental section). Under these conditions, the 1-D product has diameters in the region of about $50 \mathrm{~nm}$, and the growth essentially follows route (b) of Fig. 1. As displayed in Figs. 2(a) and 2(b), concave growing heads of nanorods along the [0001] direction can be observed in a large proportion of the product. Interestingly, an inward cone-shaped space can be observed in most growing heads of the $\mathrm{ZnO}$ nanorods (Figs. 2(c)-2(e)), and growth oscillation can also be seen clearly in the ring-like contrasts of these TEM images. Accordingly, flat $\{000-1\}$ ends of these nanorods can also be seen in an equal quantity (Figs. 2(f)-2(h)). Such oscillating crystal growth is normally associated with a periodic change in experimental parameters (such as temperature, pressure, etc.) and/or a periodic consumption of reactants and burst of products [28]. In the current case, the observed oscillation is probably an example of the latter, because the large heat-capacity autoclave used in this work is rather insensitive to momentary heating instability. Our XRD investigation reported in Fig. 3(a) indicates the $\mathrm{ZnO}$ nanorods adopt a pure wurtzite structure ( $\mathrm{Pb}_{3} \mathrm{mc}$, JCPDS card No. 36-1451) [21-27], with the measured lattice constants $c_{0}$ and $a_{0}$ of this hexagonal phase being $5.21 \AA$, and $3.25 \AA$ 


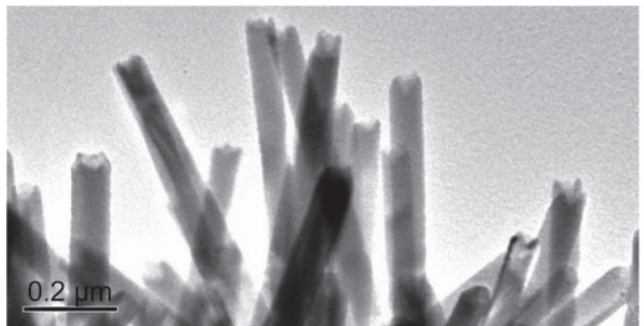

(a)

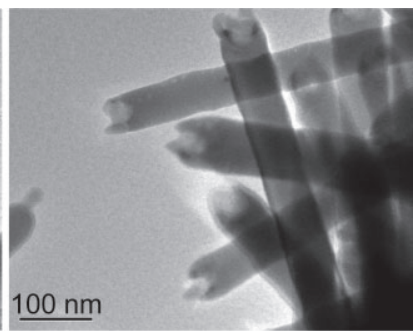

(b)

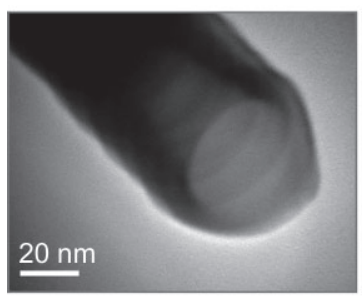

(c)

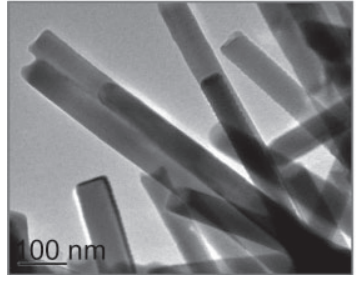

(f)

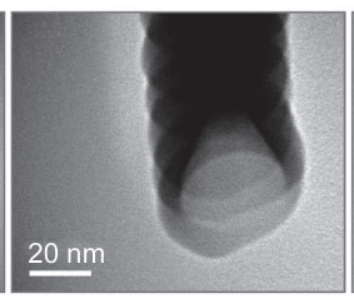

(d)

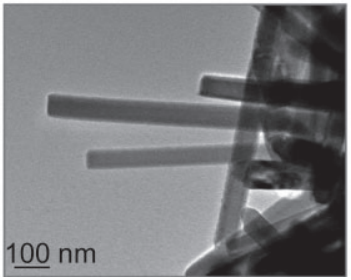

(g)

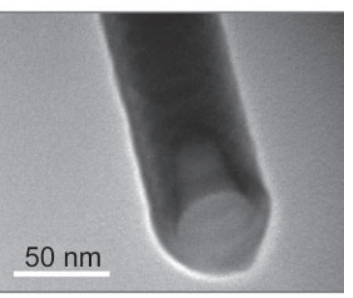

(e)

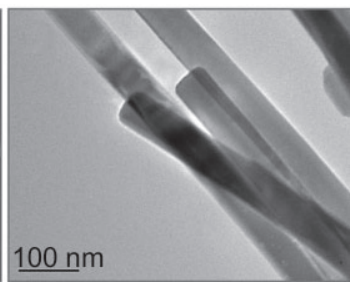

(h)

Figure 2 TEM images of the $50 \mathrm{~nm} \mathrm{ZnO}$ nanorods: (a), (b) panoramic views; (c)-(e) detailed views of the concave growing heads of $\mathrm{ZnO}$ nanorods, where growth oscillation (ring-like marks) is also apparent; (f)-(h) flat (000-1) ends of the $\mathrm{ZnO}$ nanorods

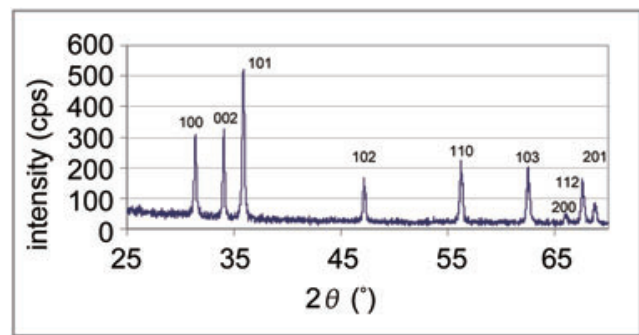

(a)

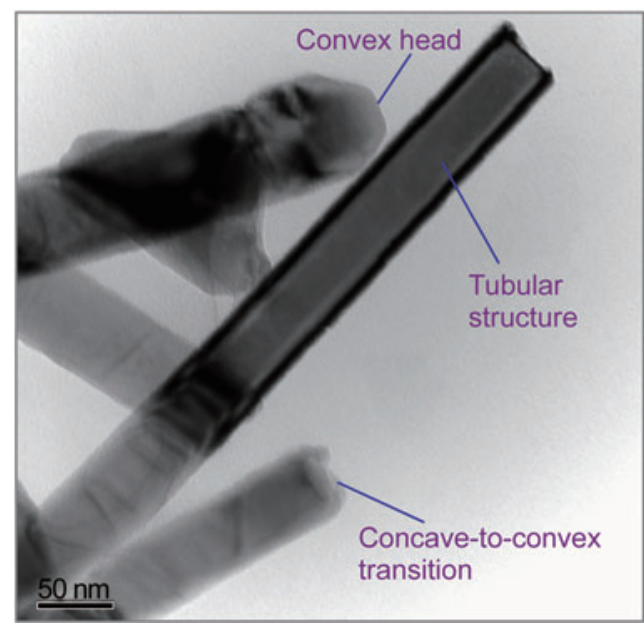

(b)

Figure 3 (a) XRD pattern of the $50 \mathrm{~nm} \mathrm{ZnO}$ nanorods, and (b) coexistence of different types of growing heads of one-dimensional $\mathrm{ZnO}$ respectively $\left(c_{0} / a_{0}=1.60\right)$. On the basis of our energy dispersive X-ray spectroscopy (EDX) data, the atomic ratio of $\mathrm{Zn}: \mathrm{O}$ in these nanorods is close to the expected value $(\approx 1: 1$; see Electronic Supplementary Material (ESM), Fig. S-1). Although the majority of the $\mathrm{ZnO}$ nanorods have concave head structures along the [0001] direction, as shown in Fig. 2, tubular openings as well as extruding tips (i.e., convex structures) can also be occasionally found within the same sample; Fig. 3(b) shows an example of the mixed situation, that is, presence of both the intermediate structure and final $\mathrm{ZnO}$ product (see Fig. 1). If the reaction time is prolonged, complete conversion to $\mathrm{ZnO}$ nanorods with convex growth fronts can be achieved, and the well faceted $\mathrm{ZnO}$ nanorods are now all in stage vi, as we have reported in an earlier investigation [24]. Taken together, our experimental observations of the different crystal morphologies confirm the existence of the proposed concave-toconvex volume conversion process (Fig. 1).

The roles of the different reagents have also been investigated (see ESM, Fig. S-2). For example, ethylenediamine acts as both a ligand to $\mathrm{Zn}^{2+}$ ions in the precursor solutions and as an adsorbate to deactivate the prismatic planes of $\mathrm{ZnO}$ [24]. An ultrasonic pretreatment of the solution proved to be essential; without it, the monodispersivity of the products was lower. Under otherwise similar experimental conditions, the $\mathrm{Zn}^{2+} / \mathrm{OH}^{-}$ratio determines the diameter of the 1-D $\mathrm{ZnO}$ products (see ESM, Fig. S-2). When the molar ratio of $\mathrm{Zn}^{2+} / \mathrm{OH}^{-}$ was increased to 1:10 (see Experimental section), the radial growth of 1-D $\mathrm{ZnO}$ was promoted, and the six prismatic planes (10-10), (01-10), (-1010), (0 $-110),(1-100)$, and $(-1100)$ became clearly defined (also see Fig. 7 later), including the slope tip region, whilst the primary growth direction was still [0001]. Typical diameters of these 1-D structures are around $400 \mathrm{~nm}$ when $\mathrm{Zn}^{2+} / \mathrm{OH}^{-}$was set to 1:10 (instead of 1:20). Surprisingly, as shown in Figs. 4 and 5, closed $\mathrm{ZnO}$ nanotubes were prepared for the first time to the reaction follows the volume inversion 
process depicted in Fig. 1. For example, open head nanotubes (Figs. 4(a) and 4(b)) can be ascribed to the crystal intermediates in stage $\mathrm{v}$, and closed nanotubes (Figs. 4(c) and 4(d)) to the final stage (vi) of this formation route. The simultaneous presence of the reaction intermediate (i.e., the open tubes)

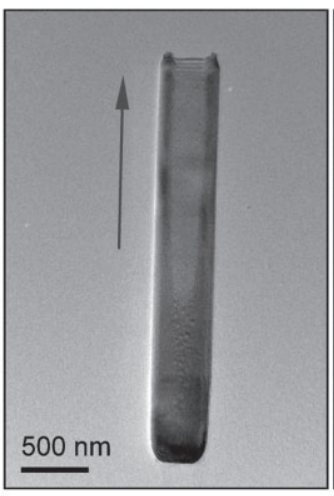

(a)

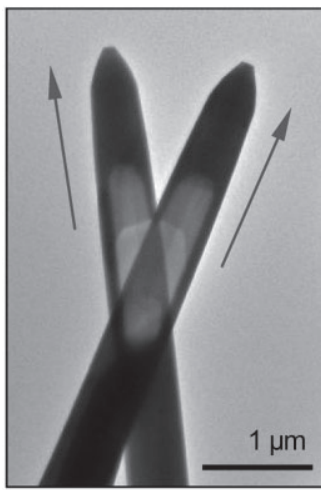

(c)

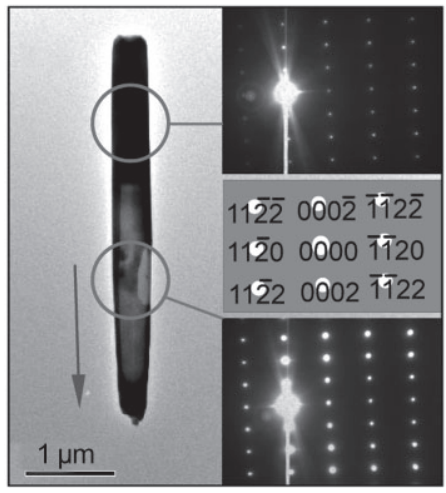

(e)

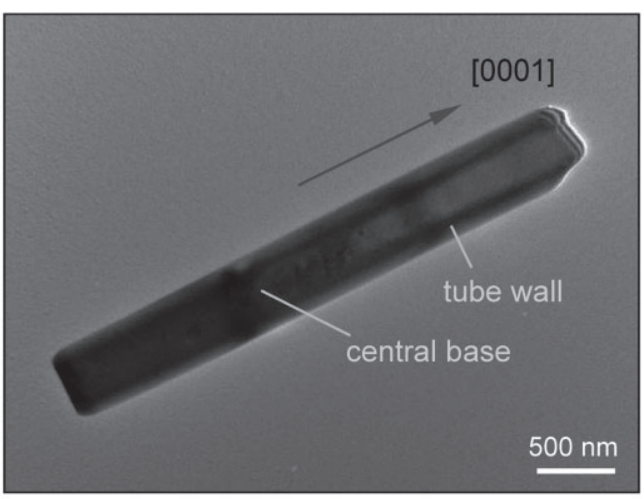

(b)

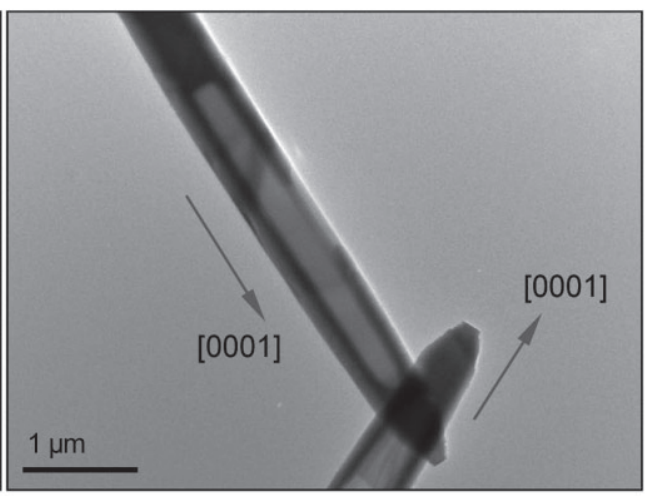

(d)

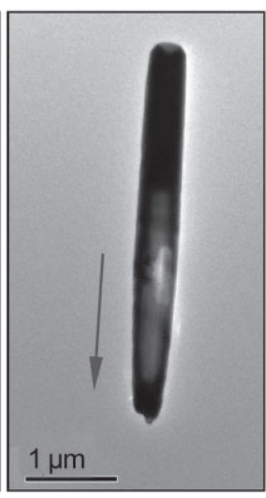

(f)

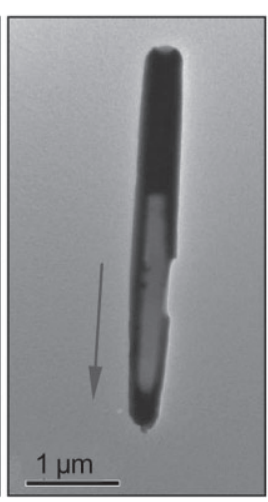

(g)
Figure 4 TEM images of the $400 \mathrm{~nm} \mathrm{ZnO}$ nanotubes: (a), (b) two concave growing heads (open ends) of $\mathrm{ZnO}$ nanotubes; (c), (d) closed $\mathrm{ZnO}$ nanotubes after volume inversion; (e)-(g) angle-dependent measurement for a closed $\mathrm{ZnO}$ nanotube $\left((\mathrm{e}) 0^{\circ},(\mathrm{f})+40^{\circ},(\mathrm{g})-40^{\circ}\right)$. Two SAED patterns and their diffraction beam assignment, which corresponds to the [1-100] zone spots of $\mathrm{ZnO}$, are also displayed in (e) for the circled areas of the nanotube. In all cases, the arrows indicate the major growth direction [0001] and the final product (i.e., the closed tubes) in the same reaction batch is good evidence in support of the growth mechanism described in Fig. 1(a). It is important to recognize that, compared to the solid nanorods of Fig. 2, the growth on the central base is much slower than that on the wall rim. After a careful examination of these nanotubes, quite interestingly, we found that for some enclosed nanotubes there was a small hole in the tube wall. Figures $4(\mathrm{e})-4(\mathrm{~g})$ show a series of TEM images of our sample tilting experiment, which illustrates the presence of this type of wall breakage. Although the reason for occurrence of the small holes is not known at this time, one plausible cause might be due to the pressure difference between entrapped liquid/vapor inside the tube cavity and the external environment of the electron microscope. It is interesting to note that the hollow $\mathrm{ZnO}$ nanotubes could also be broken into fragments (halves). The resulting fragments are normally located in adjacent positions suggesting they are probably only formed during examination under the electron microscope (see ESM, Fig. S-3), again consistent with the large pressure difference between the entrapped liquid/ vapor and the vacuum chambers of the TEM and FESEM. On the basis of our selectedarea electron diffraction (SAED, Fig. 4(e)) and high-resolution TEM studies (Fig. 5), we affirm that the nanotube materials are singlecrystalline and the axial direction is along [0001]. Furthermore, similar to the case for the $50 \mathrm{~nm} \mathrm{ZnO}$ nanorods, our structural and compositional analyses confirm that the $\mathrm{ZnO}$ nanotubes have an atomic ratio of $\mathrm{Zn}: \mathrm{O} \approx 1: 1$, and they are indeed in the wurtzite phase (Figs. 4 and 5); further details of the EDX results are given in the ESM (Fig. S-1).

In addition to the formation of the tubular structures by route (a), solid nanorods were also formed by route (b) of Fig. 1. As evidenced in Fig. 6, $\mathrm{ZnO}$ nanorods in the 400 $\mathrm{nm}$ diameter regime were also formed in the same growth experiment (population ratio: 


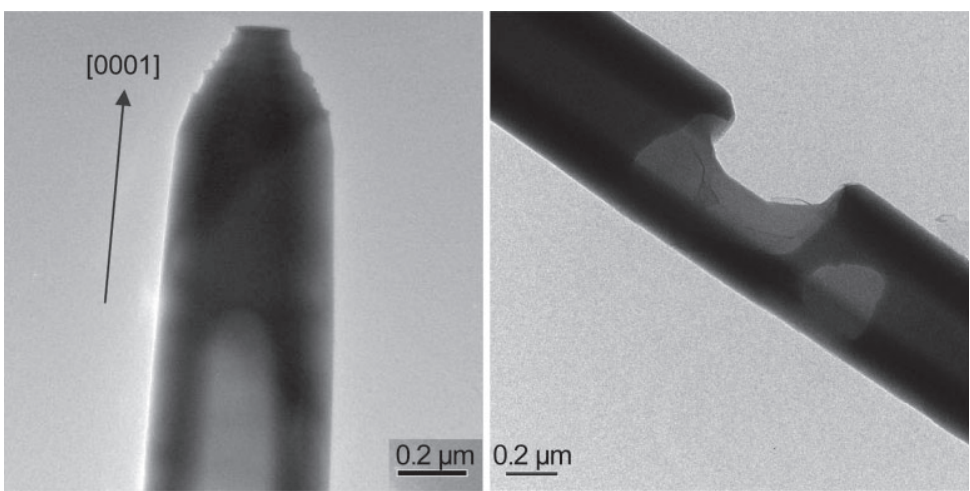

(a)

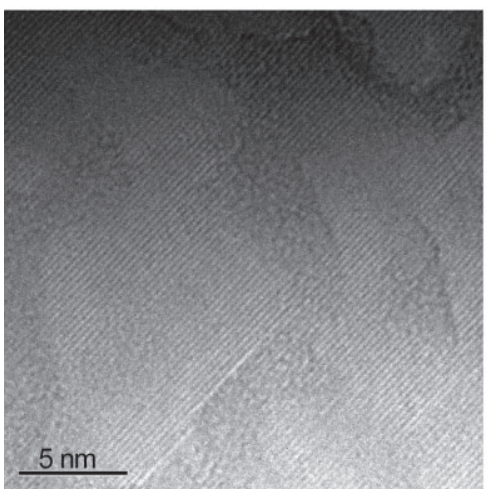

(c)

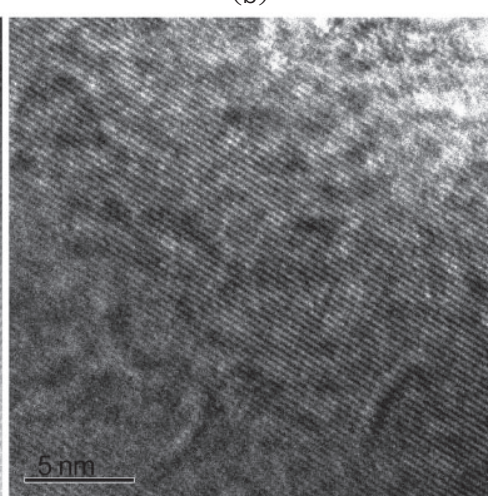

(d)

Figure 5 TEM/HRTEM images: (a) terraces in the tip slope of a $\mathrm{ZnO}$ nanotube, where six prismatic crystal planes are still stable after the volume inversion (refer to Fig. 1); (b) the wall breakage observed in a $\mathrm{ZnO}$ nanotube; (c) $d_{002}$ was determined as $2.6 \AA \pm 0.1 \AA$ from the $\mathrm{ZnO}$ nanotube in (b); (d) $d_{100}$ was determined as $2.8 \pm 0.1 \AA$ from the $\mathrm{ZnO}$ nanotube in (b)

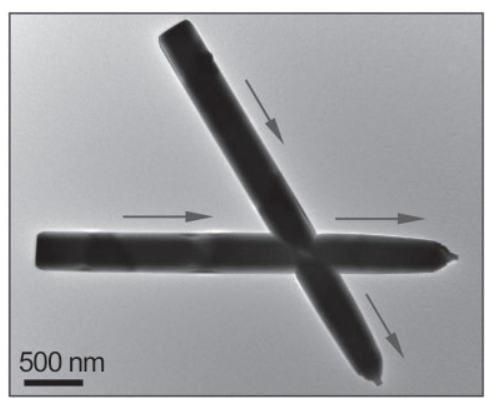

(a)

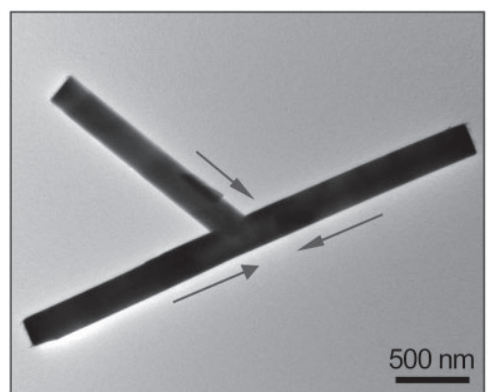

(c)

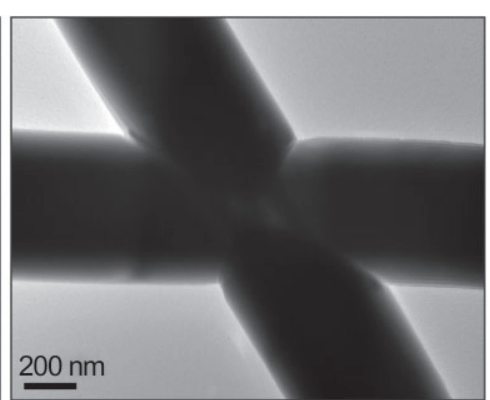

(b)

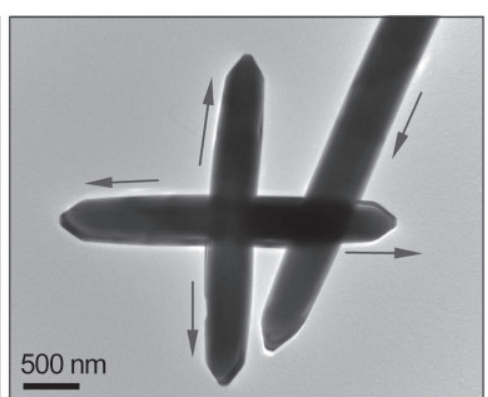

(d)
Figure 6 TEM images of the $400 \mathrm{~nm} \mathrm{ZnO}$ nanorods: (a) two intersected nanorods without branching; (b) a detailed view of the intersection of (a); (c) joining of three [0001] heads; (d) branching out of four [0001] heads (i.e., joining of four [000-1] ends. In all the cases, the arrows indicate the major growth direction [0001] nanotubes/ nanorods $\approx 35 \%: 65 \%$ ), which can be attributed to a localized variation in supersaturation due to the lack of stirring in our batch reactions (i.e., we postulate that the solid rods are formed at a lower degree of supersaturation). In contrast to their freestanding counterparts (50 $\mathrm{nm}$ nanorods) in Fig. 2 however, the larger $\mathrm{ZnO}$ nanorods are often joined together. For instance, two growing rods might intersect together while maintaining single-crystallinity in their respective continuous growths (Figs. 6(a) and $6(b))$. On the other hand, a growing rod might come into contact with other rods and terminate its own growth along the [0001] direction (Fig. 6(c)). In some cases, multiple branching along the [0001] direction of the nanorods was observed (Fig. 6(d)). All these observations seem to suggest that the [0001] growth fronts of the nanorods are highly reactive during their axial advancement. Because no net cavity was generated in the nanorods and the growth processes for their growing heads/junctures could not be confirmed explicitly (Fig. 6(b)), we will use the intersected nanotubes displayed in Fig. 7 to illustrate this point further. Compared with the single-crystal freestanding tube (Fig. 7(a)), overall assemblies of intersected $\mathrm{ZnO}$ nanotubes should be considered as polycrystals, although each individual straight segment can still be viewed as a single crystal. Similar to the solid nanorods (Fig. 6), no preferred intersection angles are revealed (Figs. $7(\mathrm{~b})$ and $7(\mathrm{c})$ ), and thus the intersection should be attributed to random encounters between the reactive growing heads. The branching of nanotubes can be further divided into two types: expanding (Figs. 7(d) and 7(e)) and terminating (Fig. 7(f)), as seen in the solid nanorods (Fig. 6). Most importantly, because the intersecting/branching takes place only in the tubular segments, it can be concluded that the concave growth fronts are highly reactive. Stereo-views of the junctures and 


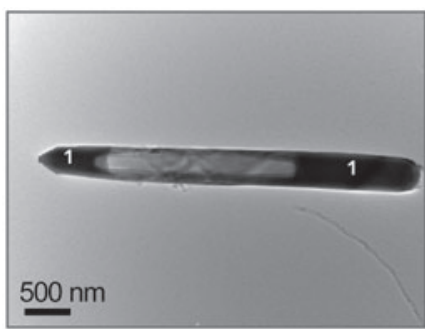

(a)

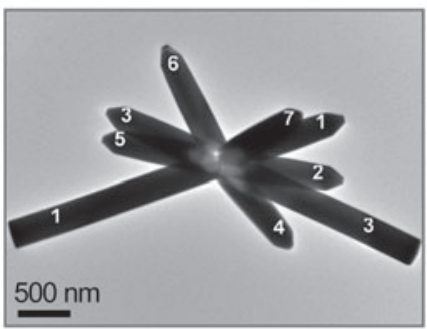

(e)

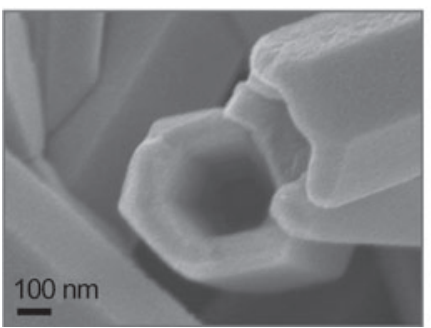

(i)

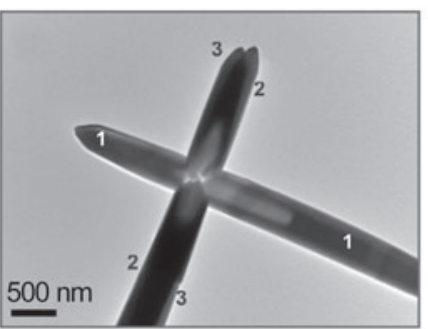

(b)

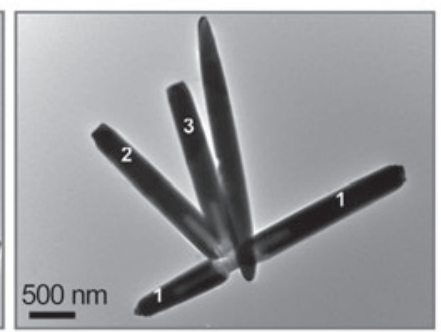

(f)

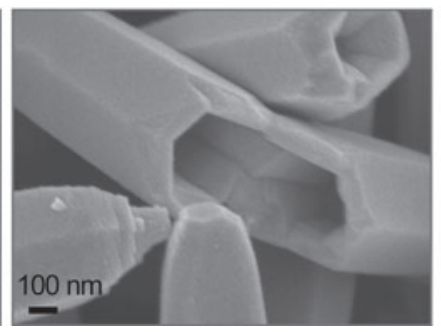

(j)

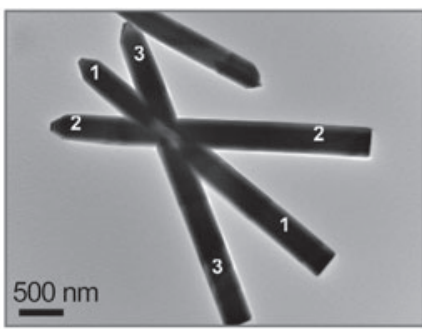

(c)

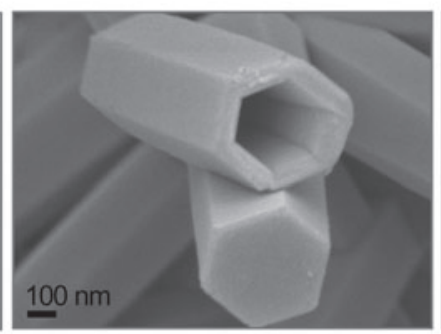

(g)

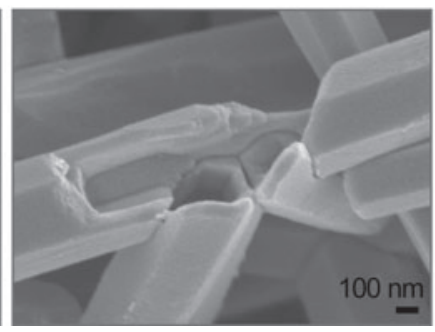

(k)

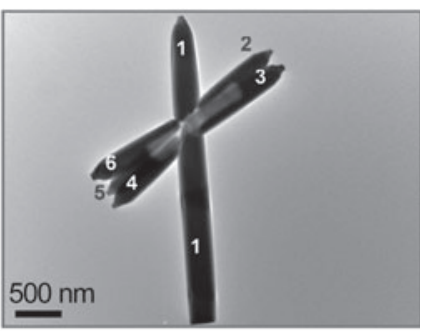

(d)

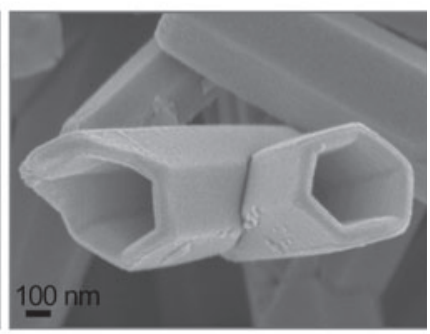

(h)

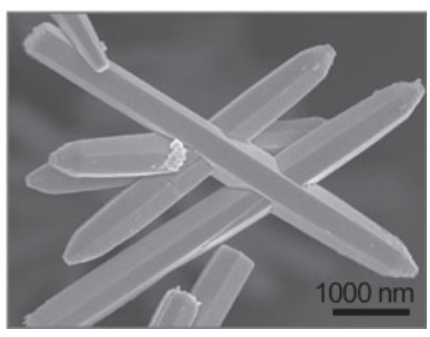

(I)

Figure 7 TEM images (a)-(f) and FESEM images (g)-(I) of the $400 \mathrm{~nm}$ ZnO nanotubes: (a) a freestanding closed nanotube; (b), (c) intersected nanotubes without branching (refer to (h), (l)); (d) a nanotube (marked 1) with five branches (refer to (i), (j)); (e) two intersected nanotubes (marked 1 and 3) with five branches (refer to (i), (j)); (f) a nanotube (marked 1) with two growing nanotubes attached (refer to (k)); ( $g$ ) nanotubes with an open head and a flat hexagonal end (i.e., (000-1) plane). In (a)-(f), the numbers serve as visual guides for individual nanotubes in the final nanotube aggregates

the hollow interiors of these intersected/branched nanotubes from our field-emission scanning electron microscopy investigation are shown in Figs. 7(g)$7(1)$.

It has been demonstrated in the above cases that a concave growing head is present during the unidirectional expansion of the $\mathrm{ZnO}$ nanomaterials. To understand why this type of head structure is adopted, we note that there are a number of advantages associated with this structure. First, it provides highly active sites for the crystal growth, because the coordination of atoms located at the rims of the $\mathrm{ZnO}$ (0001) planes is less saturated than those on the central base. Second, it enables easy access of the precursor species from solution to the growing solid, because of a shorter diffusion distance to the wall rim than to the center, including to the bottom of the cavity. Finally, it creates the largest growth front for deposition, because a concave solid-liquid interface can accommodate more incoming species owing to its larger total surface area (i.e., rim and base, inner and outer wall areas), compared to a flat (i.e., the (0001) crystal plane) or a convex liquid-solid interface which possesses only the (0001) plane and the outer crystal surface. These findings suggest that stabilization of the side crystal planes, thus creating active nucleation sites in the rim area only, is a prerequisite for the formation of a tubular structure. Furthermore, in order to prepare nanotubes a relatively high supersaturation is required during the tube formation. The volume inversion observed in this work corresponds to a change from a kinetically 
controlled to a thermodynamically controlled process. We believe that other 1-D materials that have a similar wurtzite-like crystal structure may also adopt this type of volume inversion in certain stages of their growth, although more data in this area are still required.

\section{Conclusions}

In summary, we have developed a solution approach for direct crystal growth of $\mathrm{ZnO}$ nanotubes. In addition to the preparation of single-crystalline $\mathrm{ZnO}$ nanotubes, we have also demonstrated that a concave shaped crystal growth front is generally reactive and can accommodate more depositing species during rapid expansion of unidirectional nanomaterials. Depending on the degree of supersaturation of nutrients in solution, the concave growth front can either remain unaltered or undergo a concave-toconvex transformation, leading to the growth of solid nanorods and / or hollow nanotubes. In principle, the observed volume inversion should also be applicable to nanoarchitecture of other wurtzite-like 1-D materials, although challenges remain in identifying the appropriate synthesis parameters for other target materials.

\section{Acknowledgements}

The authors gratefully acknowledge the financial support of the Ministry of Education, Singapore. B. L. would also like to thank the National University of Singapore for providing his postgraduate scholarship.

Electronic Supplementary Material: Supplementary material (details of experiments, TEM images, and EDX results) is available in the online version of this article at http://dx.doi.org/10.1007/s12274-009-9018-7 and is accessible free of charge.

\section{References}

[1] lijima, S. Helical microtubules of graphitic carbon. Nature 1991, 354, 56-58.

[2] Ajayan, P. M.; Ebbesen, T. W. Nanometre-size tubes of carbon. Rep. Prog. Phys. 1997, 60, 1025-1062.

[3] Subramoney, S. Novel nanocarbons-structure, properties, and potential applications. Adv. Mater. 1998, 10, 11571171.

[4] Ebbesen, T. W.; Ajayan, P. M. Large-scale synthesis of carbon nanotubes. Nature 1992, 358, 220-222.

[5] Thess, A.; Lee, R.; Nikolaev, P.; Dai, H.; Petit, P.; Robert, J.; Xu, C.; Lee, Y. H.; Kim, S. G.; Rinzler, A. G.; Colbert, D. T.; Scuseria, G. E.; Tomanek, D.; Fischer, J. E.; Smalley, R. E. Crystalline ropes of metallic carbon nanotubes. Science 1996, 273, 483-487.

[6] Tenne, R.; Homyonfer, M.; Feldman, Y. Nanoparticles of layered compounds with hollow cage structures (inorganic fullerene-like structures). Chem. Mater. 1998, 10, 3225-3238, and references therein.

[7] Feldman, Y.; Frey, G. L.; Homyonfer, M.; Lyakhovitskaya, V.; Margulis, L.; Cohen, H.; Hodes, G.; Hutchison, J. L.; Tenne, R. Bulk synthesis of inorganic fullerene-like $\mathrm{MS}_{2}(\mathrm{M}=\mathrm{Mo}, \mathrm{W})$ from the respective trioxides and the reaction mechanism. J. Am. Chem. Soc. 1996, 118, 5362 -5367 .

[8] Zak, A.; Feldman, Y.; Alperovich, V.; Rosentsveig, R.; Tenne, R. Growth mechanism of $\mathrm{MoS}_{2}$ fullerene-like nanoparticles by gas-phase synthesis. J. Am. Chem. Soc. 2000, 122, 11108-11116.

[9] Nath, M.; Rao, C. N. R. New metal disulfide nanotubes. J. Am. Chem. Soc. 2001, 123, 4841-4842.

[10] Vayssieres, L.; Keis, K.; Hagfeldt, A.; Lindquist, S. -E. Three-dimensional array of highly oriented crystalline ZnO microtubes. Chem. Mater. 2001, 13, 4395-4398.

[11] Li, Q.; Kumar, V.; Li, Y.; Zhang, H.; Marks, T.; Chang, R. P. H. Fabrication of $\mathrm{ZnO}$ nanorods and nanotubes in aqueous solutions. Chem. Mater. 2005, 17, 1001-1006.

[12] Tong, Y.; Liu, Y.; Shao, C.; Liu, Y.; Xu, C.; Zhang, J.; Lu, Y.; Shen, D.; Fan, X. Growth and optical properties of faceted hexagonal ZnO nanotubes. J. Phys. Chem. B. 2006, 110, 14714-14718.

[13] Tong, Y.; Liu, Y.; Dong, L.; Zhao, D.; Zhang, J.; Lu, Y.; Shen, D.; Fan, X. Growth of $\mathrm{ZnO}$ nanostructures with different morphologies by using hydrothermal technique. J. Phys. Chem. B. 2006, 110, 20263-20267.

[14] Yu, S. -Y.; Zhang, H. -J.; Peng, Z. -P.; Sun, L. -N.; Shi, W. -D. Template-free fabrication of hexagonal $\mathrm{ZnO}$ microprism with an interior space. Inorg. Chem. 2007, 46, 8019-8023.

[15] Wu, J. -J.; Liu, S. -C.; Wu, C. -T.; Chen, K. -H.; Chen, L. 
-C. Heterostructures of $\mathrm{ZnO}-\mathrm{Zn}$ coaxial nanocables and ZnO nanotubes. Appl. Phys. Lett. 2002, 81, 1312-1314.

[16] Hu, J. Q.; Li, Q.; Meng, X. M.; Lee, C. S.; Lee, S. T. Thermal reduction route to the fabrication of coaxial $\mathrm{Zn} / \mathrm{ZnO}$ nanocables and $\mathrm{ZnO}$ nanotubes. Chem. Mater. 2003, 15, 305-308.

[17] Hu, J. Q.; Bando, Y. Growth and optical properties of single crystalline ZnO whiskers. Appl. Phys. Lett. 2003 82, 1401-1403.

[18] Zhang, B. P.; Binh, N. T.; Wakatsuki, K.; Segawa, Y.; Yamada, Y.; Usami, N.; Kawasaki, M.; Koinuma, H. Formation of highly aligned $\mathrm{ZnO}$ tubes on sapphire (0001) substrates. Appl. Phys. Lett. 2004, 84, 4098-4100.

[19] Sun, Y.; Fuge, G. M.; Fox, N. A.; Riley, D. J.; Ashfold, M. N. R. Synthesis of aligned arrays of ultrathin $\mathrm{ZnO}$ nanotubes on an Si wafer coated with a thin ZnO film. Adv. Mater.

2005, 17, 2477-2481.

[20] Jeong, J. S.; Lee, J. Y.; Cho, J. H.; Suh, H. J.; Lee, C. J. Single-crystalline $\mathrm{ZnO}$ microtubes formed by coalescence of $\mathrm{ZnO}$ nanowires using a simple metal-vapor deposition method. Chem. Mater. 2005, 17, 2752-2756.

[21] Pan, Z. W.; Dai, Z. R.; Wang, Z. L. Nanobelts of semiconducting oxides. Science 2001, 291, 1947-1949.
[22] Huang, M. H.; Mao, S.; Feick, H.; Yan, H.; Wu, Y.; Kind, H.; Webber, E.; Russo, R.; Yang, P. Room-temperature ultraviolet nanowire nanolasers. Science 2001, 292, 1897-1899.

[23] Pacholski, C.; Kornowski, A.; Weller, H. Self-assembly of ZnO: From nanodots to nanorods. Angew. Chem. Int. Ed. 2002, 41, 1188-1191.

[24] Liu, B.; Zeng, H. C. Hydrothermal synthesis of ZnO nanorods in the diameter regime of $50 \mathrm{~nm}$. J. Am. Chem. Soc. 2003, 125, 4430-4431.

[25] Gao, P. X.; Ding, Y.; Mai, W.; Hughes, W. L.; Lao, C.; Wang, Z. L. Conversion of zinc oxide nanobelt into superlattice-structured nanohelices. Science 2005, 309, 1700-1704.

[26] Liu, B.; Zeng, H. C. Fabrication of ZnO "dandelions" via a modified kirkendall process. J. Am. Chem. Soc. 2004, 126, 16744-16746.

[27] Wang, Z. L.; Song, J. H. Piezoelectric nanogenerators based on zinc oxide nanowirearrays. Science 2006, 312, 242-246]

[28] Brice, J. C. Crystal Growth Processes; John Wiley and Sons: New York, 1986, Ch. 2, pp. 17-103. 